\title{
Filago ramosissima Lange (Asteraceae), novedad para la flora de Andalucía Occidental (España)
}

\section{Juan A. Devesa' \& Santiago Andrés-Sánchez ${ }^{2}$}

'Departamento de Botánica, Ecología y Fisiología Vegetal, Facultad de Ciencias, Edificio José Celestino Mutis, Campus de Rabanales, Universidad de Córdoba, 14071-Córdoba, España

2Departamento de Didáctica de la Matemática y Didáctica de las Ciencias Experimentales y Biobanco Vegetal, Banco Nacional de ADN, Universidad de Salamanca, 37008-Salamanca, España

\author{
Correspondencia \\ J. A. Devesa \\ e-mail: bv1dealj@uco.es \\ Recibido: 25 julio 2019 \\ Aceptado: 10 septiembre 2019 \\ Publicado on-line: 3 octubre 2019 \\ Editado por: B. Cabezudo
}

\begin{abstract}
Filago ramosissima Lange (Asteraceae), new record for the Western Andalusia flora (Spain)

Palabras clave: Filago, Asteraceae, flora, novedad, Andalucía Occidental, España
\end{abstract}

Key words: Filago, Asteraceae, flora, new record, Western Andalusia, Spain
Córdoba: Luque, laguna del Salobral, 30SUG96, 13-04-1987, F. García Móntoya (COFC 58211; COFC 58212).

El género Filago L. (incl. Evax Gaertn., Evacidium Pomel y Cymbolaena Smoljan.; Galbany-Casals \& al., 2010, Andrés-Sánchez et al., 2011) comprende unas 40 especies en todo el mundo, de las que 20 tienen representación en la Península Ibérica de acuerdo con la reciente revisión para Flora iberica (Andrés-Sánchez et al., 2019). Una de ellas, F. ramosissima Lange, es planta ginomonoica fácil de reconocer por su escaso tamaño, y por presentar capítulos ovoides de 2,5-3 $\mathrm{mm}$ de longitud, dispuestos en glomérulos de 1-3, a menudo los más basales solitarios, con flores femeninas y hermafroditas exiguas $(1,8-2,5$ $\mathrm{mm}$ y $1,5-3 \mathrm{~mm}$, respectivamente), y aquenios de 0,4-0,6 mm, papilosos, con el vilano integrado por 12-18 pelos.

El área de distribución de este taxón se extiende por el noroeste de África (Marruecos) y España, en donde ha sido reiteradamente citada para las provincias de Almería (Willkomm, 1865; Sagredo, 1975; Andrés-Sánchez et al., 2013) y Granada (Willkomm, 1865; Molero Mesa \& Pérez Raya, 1987; Andrés-Sánchez et al., 2013), y de manera puntual en las de Jaén (Andrés-Sánchez et al., 2013 -en anexo I-, y 2019), Murcia (Lahora et al., 2017), Zaragoza (Pike, 2003; AndrésSánchez et al., 2013) y Málaga, esta última añadida recientemente por Andrés-Sánchez et al. (2019) y basada en unos ejemplares procedentes de Ardales, Mesa de Villaverde (MGC76313). La revisión de los materiales del género conservados en el herbario COFC de la Universidad de Córdoba por uno de los autores (J. A. Devesa), ha permitido detectar la presencia de la especie en la Campiña cordobesa, basada en un material erróneamente identificado. Su presencia en Córdoba supone novedad provincial, así como para Andalucía Occidental; significa una ampliación hacia el oeste del área de este taxón mal conocido con anterioridad.

\section{Agradecimientos}

Este estudio ha sido subvencionado por el Ministerio de Ciencia e Innovación a través de los proyectos CGL2017-85204-C3-3-P y CGL2017-85204-C3-2-P, ambos cofinanciados por FEDER.

\section{Bibliografía}

Andrés-Sánchez, S., Martínez Ortega, M. M. \& Rico, E. (2019). Filago L. In: Benedí, C., Buira, A., Rico, E. \& al. (eds.), Flora Iberica, XVI(III) (pp. 1672-1705). Real Jardín Botánico (CSIC), Madrid.

Andrés-Sánchez,S., Galbany-Casals, M., Rico, E. \& Martínez-Ortega, M. M. (2011). A nomenclatural treatment for Logfia Cass. and Filago L. (Asteraceae) as newly circumscribed: Typification of several names. Taxon, 60: 572-576.

Andrés-Sánchez, S., Martínez-Ortega, M. M. \& Rico, E. (2013). Estudio corológico del género Filago L. (Asteraceae, Gnaphalieae) en la Península Ibérica y Baleares. Botanica Complutensis, 37: 57-78.

Galbany-Casals, M., Andrés-Sánchez, S., GarciaJacas, N., Susanna, A., Rico, E. \& Martínez-Ortega, M. M. (2010). How many of Cassini anagrams should 
there be? Molecular systematics and phylogenetic relationships in the Filago group (Asteraceae, Gnaphalieae), with special focus on the genus Filago. Taxon, 59: 1671-1689.

Lahora, A., Sánchez-Gómez, P., Cánovas, J. L., Jiménez, J. F. \& Mota J. F. (2017). Sobre la presencia de Filago ramosissima Lange y Fumaria munbyi Boiss. \& Reut. en la Región de Murcia. Anales de Biología 39: 99-101.

Molero Mesa, J, \& Pérez Raya, F. (1987). La flora de Sierra Nevada. Universidad de Granada. Excma. Dip.
Prov. Granada.

Pike, S. (2003). Catálogo florístico de las plantas vasculares de Zaragoza. Publicaciones del Consejo de Protección de la Naturaleza de Aragón.

Sagredo, R. (1975). Contribución al conocimiento de la flora almeriense. Anales del Instituto Botánico A. J. Cavanilles, 32: 309-321.

Willkomm, H. M. (1865). Filago. In: Willkomm, H. M. \& Lange, J. (eds.), Prodromus florae Hispanicae 2 (pp. 53-57). Stuttgart. 